\title{
The sugar beet gene encoding the sodium/proton exchanger 1 (BvNHX1) is regulated by a MYB transcription factor
}

\author{
Guy Adler · Eduardo Blumwald · Dudy Bar-Zvi
}

Received: 14 January 2010 / Accepted: 24 March 2010 / Published online: 14 April 2010

(C) The Author(s) 2010. This article is published with open access at Springerlink.com

\begin{abstract}
Sodium/proton exchangers (NHX) are key players in the plant response to salinity and have a central role in establishing ion homeostasis. NHXs can be localized in the tonoplast or plasma membranes, where they exchange sodium ions for protons, resulting in sodium ions being removed from the cytosol into the vacuole or extracellular space. The expression of most plant $N H X$ genes is modulated by exposure of the organisms to salt stress or water stress. We explored the regulation of the vacuolar NHXI gene from the salt-tolerant sugar beet plant (BvNHX1) using Arabidopsis plants transformed with an array of constructs of $B v H N X 1: \because G U S$, and the expression patterns were characterized using histological and quantitative assays. The 5' UTR of $B v N H X 1$, including its intron, does not modulate the activity of the promoter. Serial deletions show that a $337 \mathrm{bp}$ promoter fragment sufficed for driving activity that indistinguishable from that of the fulllength $(2,464 \mathrm{bp})$ promoter. Mutating four putative cis-acting elements within the $337 \mathrm{bp}$ promoter fragment revealed that MYB transcription factor(s) are involved in the activation of the expression of $B v N H X 1$ upon exposure to salt and water stresses. Gel mobility shift assay confirmed that the WT but not the mutated MYB binding site is bound by nuclear protein extracted from salt-stressed Beta vulgaris leaves.
\end{abstract}

\footnotetext{
G. Adler · D. Bar-Zvi (ه)

Department of Life Sciences,

The Doris and Bertie Black Center for Bioenergetics

in Life Sciences, Ben-Gurion University of the Negev,

84105 Beer-Sheva, Israel

e-mail: barzvi@bgu.ac.il

E. Blumwald

Department of Plant Sciences,

University of California, Davis, CA 95616, USA
}

Keywords Abiotic stress $\cdot$ Arabidopsis $\cdot$ Beta vulgaris . MYB $\cdot$ NHX $\cdot$ Promoter activity $\cdot$ Sodium/proton exchanger $\cdot$ Sugar beet

\begin{tabular}{ll}
\multicolumn{2}{l}{ Abbreviations } \\
AtNHX1 & Arabidopsis NHX1 \\
BvNHX1 & Sugar beet NHX1 \\
EMSA & Electrophoretic mobility shift assay \\
GUS & $\beta$-Glucoronidase \\
MS & Murashige and Skoog basal plant medium \\
MYB & Myeloblastosis viral oncogene homolog \\
MYC & Myelocytomatosis viral oncogene homolog \\
NHX1 & Sodium/proton exchanger 1
\end{tabular}

\section{Introduction}

Salinity and drought are the major abiotic stresses limiting the production of crop plants worldwide (Zhu 2001; Munns 2002). Plants exposed to these stress conditions respond in biochemical, physiological and molecular levels to establish a new homeostasis that would enable them to survive the stress condition (Hasegawa et al. 2000; Serrano and Rodriguez-Navarro 2001; Zhu 2001, 2003; Bohnert et al. 2006; Pardo et al. 2006; Sahi et al. 2006). Sodium/proton exchangers (NHX) are major players in maintaining ion homeostasis (Blumwald 2000; Serrano and RodriguezNavarro 2001; Pardo et al. 2006; Sahi et al. 2006). These membrane-bound antiporters utilize electrochemical gradient of protons across the tonoplast or plasma membrane to transport sodium or potassium ions into the vacuole or sodium ions outside the cell, respectively (Blumwald 2000; Serrano and Rodriguez-Navarro 2001; Zhu 2003). The overexpression of tonoplast NHXs was shown to improve plant salt tolerance (Yamaguchi and Blumwald 2005; Apse 
and Blumwald 2007). In agreement with this finding, decreased activity of tonoplast NHX resulted in increased salt sensitivity (Apse et al. 2003; Sottosanto et al. 2007).

Vacuolar NHXs were cloned from a number of glycophilic and halophilic plant species (Blumwald and Poole 1985; Barkla et al. 1995; Fukuda et al. 1998; Hamada et al. 2001; Yamaguchi et al. 2001; Xia et al. 2002; Aharon et al. 2003; Wu et al. 2004; Kagami and Suzuki 2005; Vasekina et al. 2005; Yang et al. 2005; Jiu et al. 2007; Zhang et al. 2008). It was suggested that the activity of vacuolar $\mathrm{Na}^{+} / \mathrm{H}^{+}$ antiporters differ in salt-tolerant and salt-sensitive plants. In agreement with this notion, following salt stress the induction of NHX genes is higher in salt-tolerant plants than in salt-sensitive plants (Chauhan et al. 2000; Hamada et al. 2001; Shi and Zhu 2002; Xia et al. 2002; Yokoi et al. 2002; Zhang et al. 2008). In addition, comparison of the transcriptomes of salt-sensitive Arabidopsis thaliana with that of the related tolerant species Thellungiella halophila (salsuginea) (Taji et al. 2004; Gong et al. 2005; Wong et al. 2006) suggested that these plants mainly differ in the expression levels and expression patterns of stress-related genes and ion transporters.

A large number of regulatory genes are involved in signaling pathways that are responsive to abiotic stresses (Chen and Zhu 2004). Salt and water stresses involve a number of signaling pathways, some of which are ABA dependent (Hasegawa et al. 2000; Zhu 2002; Chen and Zhu 2004; Yamaguchi-Shinozaki and Shinozaki 2006). A large number of transcription factors have been shown to be associated in the regulation of expression of target stressinducible genes. Osmotic stress signaling pathway involves the AREB/ABF and DREB2 transcription factors, which bind the ABRE/CE and DRE/CRT cis-acting elements, respectively (reviewed by Zhu 2002; Yamaguchi-Shinozaki and Shinozaki 2006). Other transcription factors such as MYB, MYC, NAC and AP2/ERF were also shown to be involved in the regulation of genes activity by these abiotic stresses (Zhu 2002; Yamaguchi-Shinozaki and Shinozaki 2006).

Tonoplasts of the salt-tolerant sugar beet (Beta vulgaris) plant were shown to possess high $\mathrm{Na}^{+} / \mathrm{H}^{+}$-exchange activity (Blumwald et al. 1987; Blumwald and Poole 1987; Pantoja et al. 1990). NHX activity was correlated with the salinity tolerance of $B$. vulgaris cell cultures (Blumwald and Poole 1987). A cDNA encoding BvNHX1 was isolated (Xia et al. 2002). The BvNHX1 transcript levels in both the suspension cell culture and whole plants increased following salt treatment and this increase was concomitant with elevated BvNHX1 protein and vacuolar $\mathrm{Na}^{+} / \mathrm{H}^{+}$antiporter activity.

Here, the promoter of $B v N H X l$ was cloned and its activity was studied in transgenic Arabidopsis expressing the $B v N H X 1:: G U S$ construct. Interestingly, the promoter of $B v N H X 1$ does not contain ABRE or DRE cis-acting ele- ments, common in promoters of osmotic stress regulated genes. The minimal 337 bp promoter sequence that was upregulated by salt treatment and osmotic stress was identified by constructs containing serial deletions of the promoter. A number of potential cis-acting element sequences were mutated to evaluate their possible role in stress-modulated expression. Of these mutations only that in the binding site for the MYB transcription factor abolished the salt stress and water stress response.

\section{Materials and methods}

\section{Cloning of the $B v N H X 1$ promoter}

Sugar beet genomic DNA was digested with DraI, EcoRV, $P v u \mathrm{II}$, or StuI and ligated to GenomeWalker ${ }^{\mathrm{TM}}$ adapter using the Clontech GenomeWalker ${ }^{\mathrm{TM}}$ Universal Kit (Clontech, Mountain View, CA, USA). Screening for BvNHXI upstream sequences was performed using general primer and gene-specific primers according to the protocol in the instructions manual. Two steps of DNA walking were performed. For the first step, the isolation of genomic DNA fragment containing partial promoter sequence and 5' UTR, the primers used were: ptH, GCGAAGCTTCGACGGCCC GGGCTG; ptN AACTGCTCCACCATGGCTTCACAT AC. The primers used for the second step (cloning of distal promoter) were: GSP6-5, CCCAGAAACCCAAGTTACA GAAAAG; BvP1, CAAGGCCCAATGCAAGTGACAAA TG; BvP2, GTGTGGAGGAGAGAGAGTCGTGTTG.

Construction of promoter serial deletions

Deletions were prepared by PCR using a combination of either $\mathrm{Bv}$-ShortEcoRI and each of the five primers designated $\mathrm{Bv}+\mathrm{NNNBam} \mathrm{HI}$ (where NNN stands for the primer position), or Bv-Long $E c o$ RI and each of the five $\mathrm{Bv}+\mathrm{NNN}$ BamHI primers, or the primer $\mathrm{Bv}+\mathrm{UTRB}$ BmHI. PCR was done using the Takara EX-Taq. Products were isolated from gels, digested with EcoRI and BamHI, and subcloned into the respective sites of the pCAMBIA $1391 \mathrm{Z}$ vector. The serial deletions forward primers were: $\mathrm{Bv}+1 \mathrm{Bam} \mathrm{HI}$, ATTGGATCCTCATGTCGAAGAGGACAAT; $\mathrm{Bv}+271$ BamHI, ATTGGATCCTCTATGGTGCCCTGTATG; Bv+ 535BamHI, ATTGGATCCGTGCATATTTATGATGAGA TAG; Bv+803BamHI, ATTGGATCCACAGACATACAT GCATCTG; Bv+1042BamHI, ATTGGATCCGAGACG GTCTAATTTTAGTAC; Bv+UTRBamHI, ATTGGATC CACGTCTGCCATAATTTTG. The serial deletions reverse primers were: $\mathrm{Bv}$-ShortEcoRI, CCAGAATTCAGT AGAAAAATCAGGAAATAG; Bv-LongEcoRI, CCAGA ATTCACACCAAACAACCACAAT for the cloning promoter sequences without or with 5' UTR, respectively. 
Site-directed mutagenesis

Site-directed mutagenesis of promoter sequences was performed using the QuikChange II ${ }^{\circledR}$ Site-Directed Mutagenesis Kit protocol (Stratagene, La Jolla, CA, USA) employing the following mutation-containing primer pairs (mutation in bold): MYB 1AT, CTTAAAAATTATTGGATTAATT CTCTTCTTACTCGAGAATTGCATTAAACTAATCAC and GTGATTAGTTTAATGCAATTCTCGAGTAAGA AGAGAATTAATCCAATAATTTTTAAG; MYB ST1, CCAAAAAAAAAAAAAATCACTAAAAAATGGATCC ATTCATTATTCGATTAATTCTCTTTC and GAAAGA GAATTAATCGAATAATGAATGGATCCATTTTTTA GTGATTTTTTTTTTTTTTGG; MYC, GGATTAATTCT CTTCTTAATAACTCTAGACATTAAACTAATCACTA AAAAATTTTTG and CAAAAATTTTTTAGTGATTA GTTTAATGTCTAGAGTTATTAAGAAGAGAATTAA TCC; napA, TAAAAATAAACGATTTTTTATAAGAC CTCGAGTTTCCTTTTATATTCCTTCTGC and GCAG AAGGAATATAAAAGGAAACTCGAGGTCTTATAA AAAATCGTTTATTTTTA. The mutated sequences were designed to contain a unique restriction site to assist in the selection of plasmids containing mutated sequences. First screening was performed by PCR amplification of the promoter sequence using $\mathrm{Bv}$-ShortEcoRI and $\mathrm{Bv}+271 \mathrm{BamHI}$ primers (see above), followed by digestion with the respective restriction enzyme. Mutations were further confirmed by DNA sequencing.

Plant material and transformation

Arabidopsis thaliana (Columbia; Arabidopsis Biological Resource Center, Columbus, OH, USA) were grown at $25^{\circ} \mathrm{C}$ and $50 \%$ humidity under a $12 \mathrm{~h} \mathrm{light} / 12 \mathrm{~h}$ dark regime. The plants were grown either in pots or in Petri dishes containing $0.5 \times$ MS medium solidified by $0.7 \%$ agar. Transformation vectors containing the respective constructs were introduced into Agrobacterium GV3101 cells, and used for genetic transformation of Arabidopsis (Clough and Bent 1998). Homozygous T2 and T3 generation transformed plants with a single transgene insert each were selected on hygromycin and used in this study. Beta vulgaris seeds (Genesis seeds Ltd., Rehovot, Israel) were sown in potting mix and were grown at $28^{\circ} \mathrm{C}$ and $50 \%$ humidity under a $16 \mathrm{~h}$ light $/ 8 \mathrm{~h}$ dark regime.

\section{GUS activity}

Histochemical staining of GUS was performed essentially as described (Jefferson 1987). Quantitative GUS activity was assayed spectrophotometrically using $p$-nitrophenyl$\beta$-D-glucuronide (Duchefa Biochemie, Haarlem, The Netherlands) (Jefferson 1987).
Electrophoretic mobility shift assay (EMSA)

Four-week-old seedlings were treated with $0.2 \mathrm{M} \mathrm{NaCl}$ for $24 \mathrm{~h}$. Leaves were harvested, nuclei and nuclear proteins were prepared as previously described (Giuliano et al. 1988). EMSA was preformed as described (Shkolnik and Bar-Zvi 2008). Mixtures containing $2.4 \mu \mathrm{g}$ of each forward and reverse oligonucleotides containing WT (ggTAACCA tataTAACCAaaTAACCAatttaaTAACCA, ggTGGTTAtta aatTGGTTAttTGGTTAtataTGGTTA) or mutated (ggCTC GAGtataCTCGAGaaCTCGAGatttaaCTCGAG, ggCTCG AGttaaatCTCGAGttCTCGAGtataCTCGAG) MYB binding sites were annealed by heating for $100^{\circ} \mathrm{C}$ and slowly cooling to RT. WT and mutated MYB binding sites are shown in CAPS, and linkers in small letters. Annealed oligonucleotides were end-labeled using Klenow fragment of DNA polymerase I and $\left[\alpha{ }^{-32} \mathrm{P}\right] \mathrm{dCTP}$. Free nucleotides were removed by gel filtration, and the resulting labeled dsDNAs were used as probes for EMSA. Nuclear proteins $(1.2 \mu \mathrm{g})$ were preincubated at RT for $10 \mathrm{~min}$ in a reaction mix contained in a final volume of $20 \mu \mathrm{l}: 20 \mathrm{mM}$ Hepes $\mathrm{KOH} \mathrm{pH} 8$, $50 \mathrm{mM} \mathrm{KCl}, 0.5 \mathrm{mM}$ EDTA, 5\% glycerol, $12.5 \mathrm{ng}$ poly(dIdC) poly (dI-dC) (GE Healthcare, Piscataway, NJ, USA). End-labeled DNA probe $(1 \mu \mathrm{l}, 3,000 \mathrm{cpm})$ was added, and mix was incubated for an additional $20 \mathrm{~min}$. Reaction mixes were loaded onto $5 \%$ polyacrylamide gels containing $0.25 \times$ TBE buffer $(22.25 \mathrm{mM}$ Tris-borate, $0.5 \mathrm{mM}$ EDTA). Samples were electrophoresed at $30 \mathrm{~V}$, gels were dried and exposed to X-ray film.

\section{Results}

Cloning of the $B v N H X 1$ promoter

GenomeWalker B. vulgaris libraries were screened for upstream sequences using a gene-specific primer, designed from the 5' UTR sequences of BvNHXI cDNA (Xia et al. 2002) and a $370 \mathrm{bp}$ upstream of the translation start ATG start codon sequence was localized. The first amplification round yielded a $704 \mathrm{bp}$ fragment. To clone the DNA sequence comprised 704 bp promoter DNA fragment and the $5^{\prime}$ UTR, genomic DNA was used as a template for the amplification using $\mathrm{ptH}$ and $\mathrm{ptN}$ primers, corresponding to the upstream and downstream sequences of this DNA fragment, respectively. PCR reactions using genomic DNA template and $\mathrm{ptH}$ and $\mathrm{ptN}$ primers to amplify the DNA sequence of the $704 \mathrm{bp}$ promoter fragment cloned in the first round of gene walking and 5' UTR GeneWalking using ptN and GSP6-5 resulted in a DNA fragment corresponding to sequences 1 and upstream of the translation start codon, respectively. The expected size of the PCR product was $1,073 \mathrm{bp}$. The amplified sequence was 
$1,678 \mathrm{bp}$ long and sequencing analysis revealed that the DNA encoding the 5' UTR of the BvNHX1 gene is interrupted by a 605 -bp long intron.

A second round of walking was preformed using the gene-specific primers $\mathrm{BvP} 1$ and $\mathrm{BvP} 2$ which were designed from sequences in the $704 \mathrm{bp}$ fragment and yielded a 911 bp DNA fragment. The final combined promoter sequence comprised 2,464 bp upstream the translation start codon, composed of 1,378 bp promoter, $304 \mathrm{bp} \mathrm{5'}$ UTR exon I, 605 bp intron and 200 bp 5' UTR exon II (Fig. 1a).

\section{Expression pattern of the $B v N H X I$ gene}

To study the expression pattern of $B v N H X 1$, we produced transgenic Arabidopsis expressing the GUS reporter gene driven by the 2,464 bp $B v N H X I$ sequence starting from $23 \mathrm{bp}$ upstream of the translation start codon (construct $L 1:: G U S)$. Histochemical GUS staining showed that the promoter was active in all vegetative tissues (Fig. 1b-g). In addition, high expression levels were detected in hydathodes and trichomes (Fig. 1c, d). The promoter was not highly active in guard cells and the roots showed a higher staining intensity at the tips and elongation zones (Fig. 1e). The $B v N H X 1$ promoter was expressed in root hairs (Fig. 1f) but was not active in emerging lateral roots (Fig. 1g). Sepals and anthers were stained, whereas petals and carpels were not (Fig. 1h). Expression in pedicels was detected at the fruit stage but not at the flower stage (Fig. 1h, i).

a
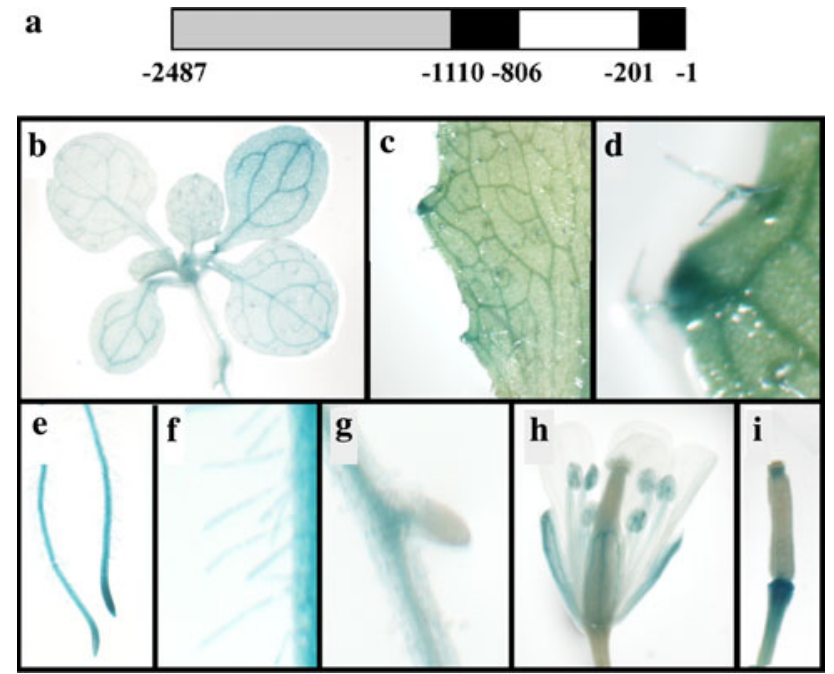

Fig. 1 Expression pattern of the full-size $B v N H X 1$ promoter. a Scheme of full-size $B v N H X 1$ promoter structure. Upstream promoter sequence, $5^{\prime}$ UTR, and intron are marked in gray, black and white, respectively. Nucleotides are numbered in according to the translation start ATG start sequence. b-i Arabidopsis plants expressing the 2,487 bp BvNHX1:: GUS constructs were grown under non-stressed conditions. Histochemical staining of GUS was performed as described in "Materials and methods". b Shoot of soil grown plant. c Section of a mature leaf. d Close-up of the edge of the leaf shown in (c). e Primary roots. $\mathbf{f}$ Root hairs. $g$ Emerging lateral root. h Flower. i Silique
To study the salt-induced expression of the BvNHXI promoter, seeds expressing the $L 1:: G U S$ construct (Fig. 2a) were germinated in the presence of $50 \mathrm{mM} \mathrm{NaCl}$. Seedlings were harvested 2 weeks later, and GUS activity was determined (Fig. 2b, c). The exposure of the seedlings to $50 \mathrm{mM}$ $\mathrm{NaCl}$ did not affect seedling growth, but resulted in a marked increase in promoter activity. The expression levels observed with this physiologically relevant long-term mild stress were much higher than those obtained with a brief exposure of non-stressed seedlings to high-salt $(>0.25 \mathrm{M})$ concentrations (not shown) according to the stress application protocol used by others (e.g., Shi and $\mathrm{Zhu}$ 2002). $\mathrm{Na}_{2} \mathrm{SO}_{4}$ and mannitol resulted in similar levels of GUS activation to that obtained using $\mathrm{NaCl}$ (Fig. 2c), suggesting that the activity can also be induced by osmotic stress. Interestingly, a similar concentration of $\mathrm{KCl}$ resulted in higher expression rates. ABA, on the other hand, had a slight, statistically non-significant increase in GUS activity. The various mild stress treatments resulted in less than $30 \%$ differences in the size of the seedlings.

Interestingly, similar to DNA sequence encoding the $5^{\prime}$ UTR of the Arabidopsis AtNHX1, the 5' UTR of BvNHXl also contains an intron. To explore whether the 5' UTR and intron play a role in the transcription regulation of the BvNHX1 gene, we transformed Arabidopsis plants with constructs in which the promoter sequence upstream of the transcription start $(S 1:: G U S)$ or containing the $5^{\prime}$ UTR + intron (5' UTR::GUS) were fused with GUS (Fig. 2a), and the plants were tested for GUS activity. GUS activities assayed in plants transformed with $L 1:: G U S$ and S1::GUS were similar and differences were not statistically significant (not shown). The expression pattern of the S1::GUS lines, containing a 1,378 bp promoter sequence upstream of the transcription start, was indistinguishable to that of plants transformed with the $L 1:: G U S$ construct both qualitatively (Fig. 2b) and quantitatively (Fig. 2c). On the other hand, the 5' UTR::GUS construct had GUS activity similar to that of non-transformed control plants (Fig. 2b, c), suggesting that this sequence is not likely to affect the transcription of this gene.

\section{Construction of serial deletions}

Five constructs comprising upstream sequences fused to reporter gene GUS were made. These constructs, designated $S 1:: G U S$ to $S 5:: G U S$, contained $1,378,1,108,844$, 576 , and $337 \mathrm{bp}$ promoter sequences upstream of the $5^{\prime}$ UTR. T2 and T3 generation plants from homozygous lines containing a single insertion were selected and at least five independent lines of each construct were tested. GUS activity in homogenates prepared from non-stressed and salttreated seedlings was determined (Fig. 3). Deletions of 270 and $534 \mathrm{bp}$ from the $5^{\prime}$ of the promoter sequence (S2 and 
a

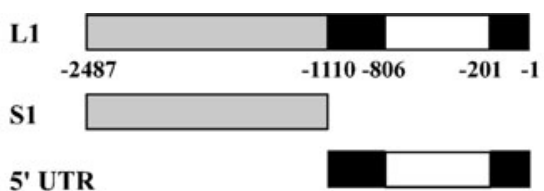

b

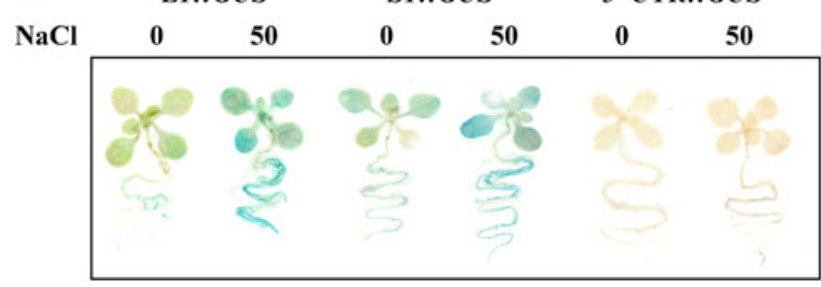

c

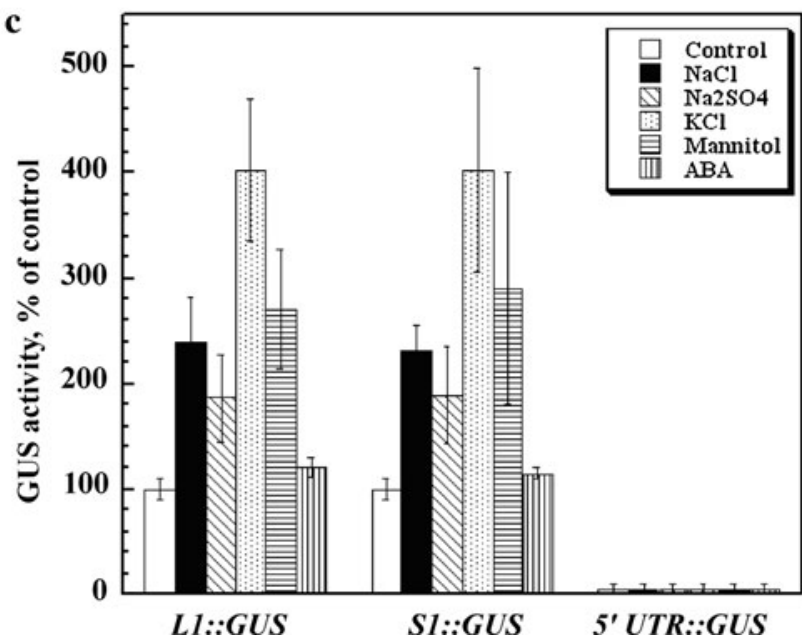

Fig. 2 Expression of constructs containing $B v N H X 1$ full-size promoter, promoter sequence upstream of the $5^{\prime}$ UTR, and 5' UTR including intron. a Constructs used. Upstream promoter sequence, 5' UTR, and intron are marked in gray, black and white, respectively. Nucleotides are numbered in according to the translation start ATG start sequence. b Histochemical staining of seedlings germinated and grown on solid medium without or with salt. Homozygous seeds of Arabidopsis transformed with the indicated constructs were sown on plates containing $0.5 \times$ MS solid medium (0) or the same medium supplemented with $50 \mathrm{mM} \mathrm{NaCl}$ (50). Two-week-old seedlings were stained for GUS. c Quantitative GUS expression in non-stressed and stressed Arabidopsis seedlings. GUS activity was assayed in homogenates of homozygous seedlings described in (b). Data shown represent average activity determined in homogenates prepared from 50 seedlings each of five independent lines of each constructs germinated and grown for 2 weeks on plates containing solid $0.5 \times$ MS medium without (white bars) or containing in addition $50 \mathrm{mM} \mathrm{NaCl}$ (black bars), $25 \mathrm{mM}$ $\mathrm{Na}_{2} \mathrm{SO}_{4}$ (diagonally dashed), $50 \mathrm{mM} \mathrm{KCl}$ (dotted), $100 \mathrm{mM}$ mannitol (horizontally dashed), or $20 \mu \mathrm{M} \mathrm{ABA} \mathrm{(vertically} \mathrm{dashed).} \mathrm{Activity} \mathrm{is}$ normalized to allow averaging between different transgenic lines and different experiments. GUS activities of $L 1:: G U S$ and $S 1:: G U S$ constructs under control conditions were similar, and were not statistically different

S3, respectively) did not affect the promoter activity. Deletion of an additional $268 \mathrm{bp}$ fragment inhibited the activation capacity of the resulting $567 \mathrm{bp}$ promoter fragment (construct S4) both in the absence and presence of salt. On the other hand, the $S 5:: G U S$ construct containing the short-

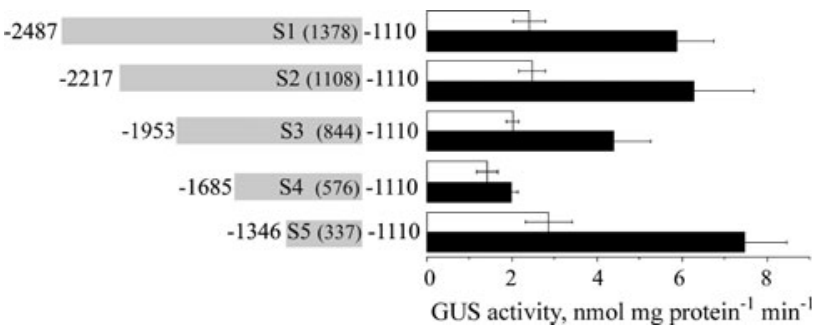

Fig. 3 Serial deletion analysis of the $B v N H X 1$ promoter activity. The $5^{\prime}$ serial deletions of the upstream promoter sequence (corresponding to the $\mathrm{S} 1$ sequence in Fig. 2) were fused to GUS and used to transform Arabidopsis. Nucleotides are numbered as in Fig. 2. GUS was assayed in homogenates prepared from 50 seedlings of each of five independent lines of each constructs, germinated and grown for 2 weeks on plates containing solid $0.5 \times$ MS medium without (white bars) or containing in addition $50 \mathrm{mM} \mathrm{NaCl}$ (black bars)

est, $337 \mathrm{bp}$ long, promoter fragment was sufficient to drive GUS expression to similar levels to that obtained by the S1::GUS construct both in the presence and absence of salt (Fig. 4). These results suggested that the transactivation capacity of the S5 promoter fragment was essentially identical to that of the full-length S1.

Analysis of potential cis-acting elements in the BvNHXI minimal promoter region

The DNA sequence of the S5 construct was used to search for potential binding sites of known trans-acting factors through the Plant cis-acting Regulatory DNA Elements (PLACE) database (Higo et al. 1999) http://www.dna. affrc.go.jp/PLACE/ (Fig. 5a). Four of these potential sites were further studied using site-directed mutagenesis (Table 1). The sequences are putative binding sites for MYB 1AT, MYC, MYB ST1, and napA transcription factors. Transcription factors from the MYC and MYB families are known to be also involved in the modulation of salt stress and water stress regulated genes (Zhu 2002; Shinozaki et al. 2003), whereas napA was shown to function in seeds (Stalberg et al. 1996). Site-directed mutagenesis was performed using Stratagene QuikChange II $^{\circledR}$, where the putative $c i s$-acting sequence was changed into a restriction enzyme recognition sequence (Table 1). Mutated sequences were confirmed by restriction digests and DNA sequence analyses, and were used for the genetic transformation of Arabidopsis plants. Homozygous transgenic plants were selected and assayed for GUS activity. The basal expression levels in non-stressed plants were not affected by the mutation of any of the potential cis-acting elements (Fig. 5b). Mutation in the MYB 1AT box (position -1362) almost abolished the induction of promoter activity by $\mathrm{NaCl}$ and mannitol, suggesting that the $B v N H X 1$ promoter is regulated mainly by a MYB $1 \mathrm{AT}$ transcription factor. Other mutations did not affect the expression of the GUS 


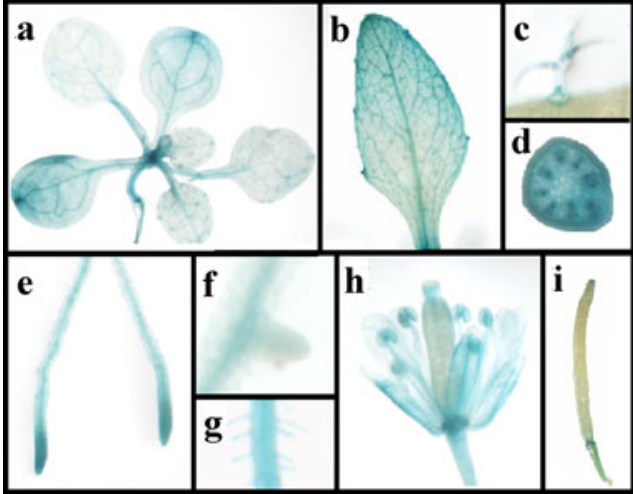

Fig. 4 Activity of the 337 bp upstream promoter fragment. Homozygous plants expressing the S5 construct (Fig. 3, 337 bp promoter fragment fused to GUS) were assayed for the activity of the reporter gene. a-i Histochemical staining of non-stressed plants. a Shoot of soil grown plant. b Mature leaf of soil grown plant. c Trichome. d Crosssection of inflorescence stem. e Roots. f Emerging lateral root. g Root

reporter gene compared to the non-mutated S5 promoter fragment.

To determine if the MYB 1AT box found in the heterologous A. thaliana species is also recognized in B. vulgaris, we challenged nuclear protein preparation extracted from salt-treated B. vulgaris seedlings for binding probes containing the WT or mutated MYB 1AT box. Figure 6 shows that $B$. vulgaris nuclear proteins bind WT MYB 1AT DNA sequence, but are incapable of binding the mutated sequence. Moreover, nuclear extract prepared from irrigated leaves did not show DNA binding activity under the conditions tested (Fig. 6) in agreement with Fig. 5.

\section{Discussion}

NHX-like sodium/proton exchangers play important roles in establishing $\mathrm{pH}$ and ion homeostasis during salt stress (Pardo et al. 2006; Apse and Blumwald 2007). It has been suggested that an important difference between NHX genes from salt-sensitive and salt-tolerant plants is not in possible differences in the gene sequences but it resides in their expression patterns and the regulation of their expression. Similar to the Arabidopsis thaliana AtNHX1, BvNHXI is expressed in leaves and roots and also in stamen, petal fruit bases and fruit tips (Shi and Zhu 2002). On the other hand, unlike AtNHX1, BvNHX1 is highly expressed in root tips and trichomes, but not in guard cells, at least in Arabidopsis (Fig. 1).

The 5' UTR of BvNHXI contains a $605 \mathrm{bp}$ intron (Fig. 1a). Introns have been shown in some cases to increase transcriptional activity in plants (Rose 2004) in a process known as intron-dependent enhancement of transcription (IME) (Rose 2008). A recent bioinformatics

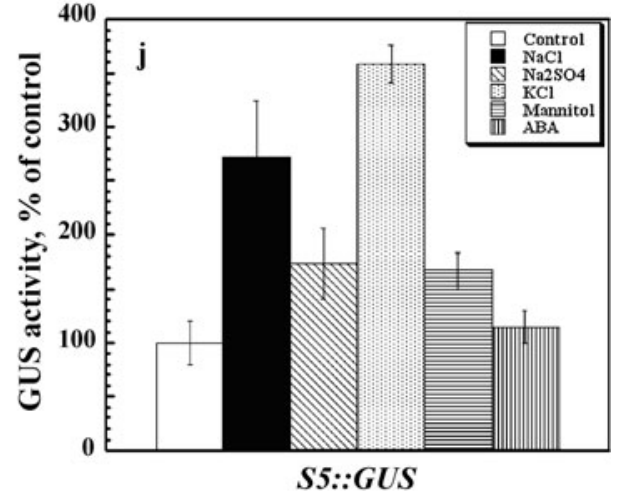

hairs. h Flower. i Silique. j Quantitative analysis using homogenates (five independent lines, 50 seedling each) from seedlings grown for 2 weeks in the $0.5 \times$ MS solid medium without (white bar) or containing in addition $50 \mathrm{mM} \mathrm{NaCl}$ (black bar), $25 \mathrm{mM} \mathrm{Na}_{2} \mathrm{SO}_{4}$ (diagonally dashed), $50 \mathrm{mM} \mathrm{KCl}$ (dotted), $100 \mathrm{mM}$ mannitol (horizontally dashed), or $20 \mu \mathrm{M} \mathrm{ABA}$ (vertically dashed)
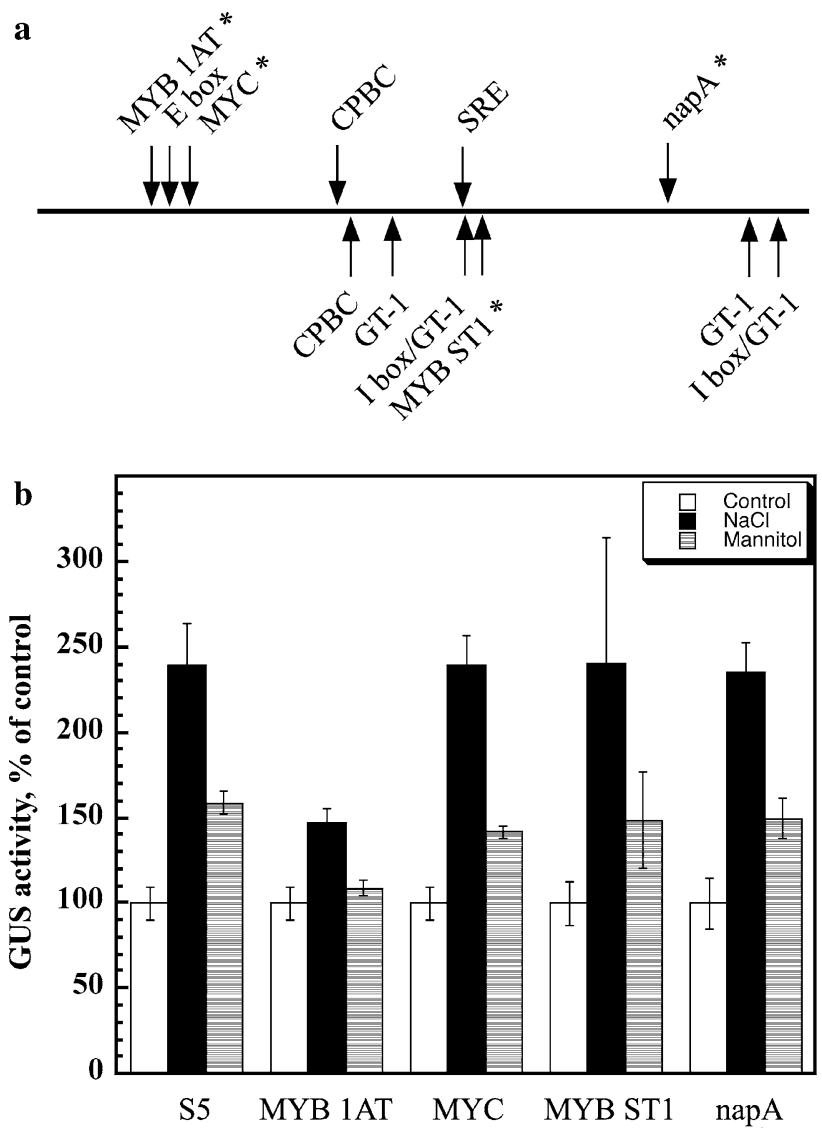

Fig. 5 Activities of mutated $337 \mathrm{bp} B v N H X 1$ promoter fragments. a Putative cis-acting elements in the S5 promoter fragment. Sites in forward or reverse modes are marked above and below the line, respectively. Mutated sequences are marked by asterisks. b GUS activity was assayed in homogenates made from five homozygous lines of Arabidopsis containing S5::GUS constructs with the indicated mutations (see also Table 1). Seeds were sowed on $0.5 \times$ MS (white bars) supplemented with $50 \mathrm{mM} \mathrm{NaCl}$ (black bars) or $100 \mathrm{mM}$ mannitol (dashed bars), and seedlings harvested and analyzed 2 weeks later 
Table 1 Mutations of putative DNA binding sites of transcription factors

\begin{tabular}{|c|c|c|c|c|c|}
\hline \multirow{2}{*}{ Recognition } & \multirow{2}{*}{ Location } & \multirow{2}{*}{ Orientation } & \multicolumn{2}{|c|}{ DNA sequence } & \multirow{2}{*}{ Restriction site } \\
\hline & & & WT & Mutated & \\
\hline MYB 1AT & -1362 & + & ATAACCA & CTCGAGA & XhoI \\
\hline MYC & -1358 & \pm & CAATTG & TCTAGA & $X b a \mathrm{I}$ \\
\hline MYB ST1 & -1228 & - & TATCCT & $\underline{\text { GGATCC }}$ & BamHI \\
\hline napA & -1136 & + & САAАСАC & CCTCGAG & XhoI \\
\hline
\end{tabular}

DNA sequences of putative DNA binding motifs (in bold) were mutagenized into a sequence containing restriction site (underlined)

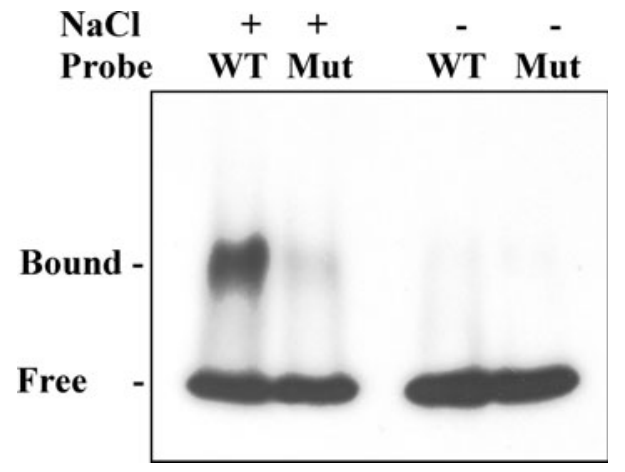

Fig. 6 B. vulgaris nuclear proteins bind WT but not mutated MYB 1 AT DNA sequence. End-labeled dsDNA probes containing WT and mutated sequence were incubated with protein nuclear extract prepared from control or salt-treated B. vulgaris leaves. Mixes were resolved by native electrophoresis on $5 \%$ polyacrylamide, the gel was dried and exposed to X-ray film. Free and Bound, migration of the free and protein bound dsDNA probe, respectively

study suggested that almost $20 \%$ of genomic sequences in Arabidopsis contain 5' UTR introns (Chung et al. 2006). The BvNHX1 5' UTR and its intron do not seem to affect the extent of gene expression, since their removal did not have any effect on the expression driven by $5^{\prime}$ upstream sequences in both the absence or presence of salt- and osmotic-stress (Fig. 2). Moreover, the DNA encoding the $5^{\prime}$ UTR and intron was not sufficient for driving expression (Fig. 2). Thus, the role of the BvNHXI 5' UTR intron remains to be determined.

Activity assays of the $5^{\prime}$ deletion series of the BvNHXI promoter suggested that nucleotides at positions -2487 to -1954 do not contain major cis-acting elements affecting gene expression (Fig. 3). On the other hand, the positions -1953 to -1686 and -1685 to -1347 might contain positive and negative elements, respectively (Fig. 3). Interestingly, a 347 bp promoter fragment $(-1346$ to -1110$)$ could drive the expression of the GUS reporter gene (Figs. 3, 4) to similar levels to that of the full-length promoter sequence (Fig. 1, 2).

Bioinformatics analysis suggested a number of potential cis-acting elements occurring within this $347 \mathrm{bp}$ promoter sequence (Fig. 5). Some of the proposed transcription factors are known to be regulated by salinity and drought.
There are two binding sites for MYB transcription factors: the MYB 1AT binding site [consensus sequence (A/ T)AACCA, TAACC in $B v N H X 1]$ in the promoters of dehydration-responsive gene $r d 22$ and many other genes (Abe et al. 2003), and the MYB ST1 (Baranowskij et al. 1994), a MYB homolog containing only one repeat that can function as a transcriptional activator. The Myb motif of the MybSt1 protein (GGATA) is distinct from the plant Myb DNA binding domain described so far. MYC (consensus sequence CANNTG, CAATTG in BvNHXI) was shown to act in promoters of genes regulated by drought and cold stress (Abe et al. 2003; Chinnusamy et al. 2004; YamaguchiShinozaki and Shinozaki 2006). The napA element conserved in many seed-expressed genes (Stalberg et al. 1996). Interestingly the $B v N H X I$ promoter lacks a binding site for ABRE, a major transcription factor regulating genes modulated by salt and water stresses (Yamaguchi-Shinozaki and Shinozaki 2005). Mutating the MYB 1AT-binding site eliminated the salt and osmotic activation of the transcription activity of $B v N H X 1$, whereas the other mutations did not affect its expression. EMSA assay using nuclear extract prepared from B. vulgaris leaves (Fig. 6) confirmed the results obtained with the heterologous $A$. thaliana plants (Fig. 5), e.g. DNA binding activity of the MYB 1AT sequence was induced by salt treatment, and was abolished when the DNA binding site was mutated. These results suggest that the mechanism is conserved between some sensitive and tolerant species. In vitro binding of the tobacco NtMYBJS1 to the AACC core motif was reduced by mutation of this sequence (Galis et al. 2006). The MYB family is the largest transcription factor family in plants with family size of up to 258 members, depending on the prediction algorithm used (Qu and Zhu 2006). The nature of the MYB protein(s) that modulate the activity of this gene is yet to be discovered. Nevertheless, this protein activity is not dependent on ABA. Expression analysis of 163 genes of the Arabidopsis MYB transcription factor family revealed that about $25 \%$ of them are affected by ABA (Yanhui et al. 2006).

The short promoter sequence identified here could be used as a water deficit-regulated promoter for driving gene expression in transgenic plants. 
Acknowledgments The authors thank Tao Xia for construction of the genomic library and the initial screen and Marina Large for technical help in preparation of DNA constructs and screening of transgenic Arabidopsis plants. Research was supported by a grant from NSFMCB-0343279. Dudy Bar-Zvi is the incumbent of The Israel and Bernard Nichunsky Chair in Desert Agriculture, Ben-Gurion University. Eduardo Blumwald is the incumbent of The Will Lester Endowed Chair in Pomology, University of California, Davis.

Open Access This article is distributed under the terms of the Creative Commons Attribution Noncommercial License which permits any noncommercial use, distribution, and reproduction in any medium, provided the original author(s) and source are credited.

\section{References}

Abe H, Urao T, Ito T, Seki M, Shinozaki K, Yamaguchi-Shinozaki K (2003) Arabidopsis AtMYC2 (bHLH) and AtMYB2 (MYB) function as transcriptional activators in abscisic acid signaling. Plant Cell 15:63-67

Aharon GS, Apse MP, Duan SL, Hua XJ, Blumwald E (2003) Characterization of a family of vacuolar $\mathrm{Na}^{+} / \mathrm{H}^{+}$antiporters in Arabidopsis thaliana. Plant Soil 253:245-256

Apse MP, Blumwald E (2007) $\mathrm{Na}^{+}$transport in plants. FEBS Lett 581:2247-2254

Apse MP, Sottosanto JB, Blumwald E (2003) Vacuolar cation $/ \mathrm{H}^{+}$exchange, ion homeostasis, and leaf development are altered in a TDNA insertional mutant of AtNHX1, the Arabidopsis vacuolar $\mathrm{Na}^{+} / \mathrm{H}^{+}$antiporter. Plant J 36:229-239

Baranowskij N, Frohberg C, Prat S, Willmitzer L (1994) A novel DNA-binding protein with homology to Myb oncoproteins containing only one repeat can function as a transcriptional activator. EMBO J 13:5383-5392

Barkla BJ, Zingarelli L, Blumwald E, Smith JAC (1995) Tonoplast $\mathrm{Na}^{+} / \mathrm{H}^{+}$antiport activity and its energization by the vacuolar $\mathrm{H}^{+}-$ ATPase in the halophytic plant Mesembryanthemum crystallinum L. Plant Physiol 109:549-556

Blumwald E (2000) Sodium transport and salt tolerance in plants. Curr Opin Cell Biol 12:431-434

Blumwald E, Poole RJ (1985) $\mathrm{Na}^{+} / \mathrm{H}^{+}$antiport in isolated tonoplast vesicles from storage tissue of Beta vulgaris. Plant Physiol 78:163-167

Blumwald E, Poole RJ (1987) Salt tolerance in suspension cultures of sugar beet-induction of $\mathrm{Na}^{+} / \mathrm{H}^{+}$antiport activity at the tonoplast by growth in salt. Plant Physiol 83:884-887

Blumwald E, Cragoe EJ, Poole RJ (1987) Inhibition of $\mathrm{Na}^{+} / \mathrm{H}^{+}$antiport activity in sugar beet tonoplast by analogs of amiloride. Plant Physiol 85:30-33

Bohnert HJ, Gong QQ, Li PH, Ma SS (2006) Unraveling abiotic stress tolerance mechanisms - getting genomics going. Curr Opin Plant Biol 9:180-188

Chauhan S, Forsthoefel N, Ran YQ, Quigley F, Nelson DE, Bohnert $\mathrm{HJ}$ (2000) $\mathrm{Na}^{+} /$myo-inositol symporters and $\mathrm{Na}^{+} / \mathrm{H}^{+}$-antiport in Mesembryanthemum crystallinum. Plant J 24:511-522

Chen WQJ, Zhu T (2004) Networks of transcription factors with roles in environmental stress response. Trends Plant Sci 9:591-596

Chinnusamy V, Schumaker K, Zhu JK (2004) Molecular genetic perspectives on cross-talk and specificity in abiotic stress signalling in plants. J Exp Bot 55:225-236

Chung BYW, Simons C, Firth AE, Brown CM, Hellens RP (2006) Effect of 5' UTR introns on gene expression in Arabidopsis thaliana. BMC Genomics 7:120

Clough SJ, Bent AF (1998) Floral dip: a simplified method for Agrobacterium-mediated transformation of Arabidopsis thaliana. Plant J 16:735-743
Fukuda A, Yazaki Y, Ishikawa T, Koike S, Tanaka Y (1998) $\mathrm{Na}^{+} / \mathrm{H}^{+}$ antiporter in tonoplast vesicles from rice roots. Plant Cell Physiol 39:196-201

Galis I, Simek P, Narisawa T, Sasaki M, Horiguchi T, Fukuda H, Matsuoka K (2006) A novel R2R3 MYB transcription factor NtMYBJS1 is a methyl jasmonate-dependent regulator of phenylpropanoid-conjugate biosynthesis in tobacco. Plant J 46:573-592

Giuliano GE, Pichersky E, Malik VS, Timko MP, Scolnik PA, Cashmore AR (1988) An evolutionarily conserved protein binding sequence upstream of a plant light-regulated gene. Proc Natl Acad Sci USA 85:7089-7093

Gong QQ, Li PH, Ma SS, Rupassara SI, Bohnert HJ (2005) Salinity stress adaptation competence in the extremophile Thellungiella halophila in comparison with its relative Arabidopsis thaliana. Plant J 44:826-839

Hamada A, Shono M, Xia T, Ohta M, Hayashi Y, Tanaka A, Hayakawa $\mathrm{T}$ (2001) Isolation and characterization of a $\mathrm{Na}^{+} / \mathrm{H}^{+}$antiporter gene from the halophyte Atriplex gmelini. Plant Mol Biol 46:3542

Hasegawa PM, Bressan RA, Zhu JK, Bohnert HJ (2000) Plant cellular and molecular responses to high salinity. Annu Rev Plant Physiol Plant Mol Biol 51:463-499

Higo K, Ugawa Y, Iwamoto M, Korenaga T (1999) Plant cis-acting regulatory DNA elements (PLACE) database: 1999. Nucl Acids Res 27:297-300

Jefferson RA (1987) Assaying chimeric genes in plants: the GUS gene fusion system. Plant Mol Biol Rep 5:387-405

Jiu JJ, Zhang XJ, Lu CF (2007) Characterization of the $\mathrm{Na}^{+} / \mathrm{H}^{+}$antiporter in tonoplast vesicles from Populus tremula calli. Prog Biochem Biophys 34:1303-1307

Kagami T, Suzuki M (2005) Molecular and functional analysis of a vacuolar $\mathrm{Na}^{+} / \mathrm{H}^{+}$antiporter gene of Rosa hybrida. Genes Genet Syst 80:121-128

Munns R (2002) Comparative physiology of salt and water stress. Plant Cell Environ 25:239-250

Pantoja O, Dainty J, Blumwald E (1990) Tonoplast ion channels from sugar-beet cell-suspensions. Inhibition by amiloride and its analogs. Plant Physiol 94:1788-1794

Pardo JM, Cubero B, Leidi EO, Quintero FJ (2006) Alkali cation exchangers: roles in cellular homeostasis and stress tolerance. J Exp Bot 57:1181-1199

Qu LJ, Zhu YX (2006) Transcription factor families in Arabidopsis: major progress and outstanding issues for future research-commentary. Curr Opin Plant Biol 9:544-549

Rose AB (2004) The effect of intron location on intron-mediated enhancement of gene expression in Arabidopsis. Plant J 40:744-751

Rose AB (2008) Nuclear pre-mRNA processing in plants. Curr Topic Microbiol Immunol 326:227-290

Sahi C, Singh A, Blumwald E, Grover A (2006) Beyond osmolytes and transporters: novel plant salt-stress tolerance-related genes from transcriptional profiling data. Physiol Plant 127:1-9

Serrano R, Rodriguez-Navarro A (2001) Ion homeostasis during salt stress in plants. Curr Opin Cell Biol 13:399-404

Shi HZ, Zhu JK (2002) Regulation of expression of the vacuolar $\mathrm{Na}^{+} /$ $\mathrm{H}^{+}$antiporter gene AtNHX1 by salt stress and abscisic acid. Plant Mol Biol 50:543-550

Shinozaki K, Yamaguchi-Shinozaki K, Seki M (2003) Regulatory network of gene expression in the drought and cold stress responses. Curr Opin Plant Biol 6:410-417

Shkolnik D, Bar-Zvi D (2008) Tomato ASR1 abrogates the response to abscisic acid and glucose in Arabidopsis by competing with ABI4 for DNA binding. Plant Biotechnol J 6:368-378

Sottosanto JB, Saranga Y, Blumwald E (2007) Impact of AtNHX1, a vacuolar $\mathrm{Na}^{+} / \mathrm{H}^{+}$antiporter, upon gene expression during shortterm and long-term salt stress in Arabidopsis thaliana. BMC Plant Biol 7:18 
Stalberg K, Ellerstom M, Ezcurra I, Ablov S, Rask L (1996) Disruption of an overlapping E-box/ABRE motif abolished high transcription of the napA storage-protein promoter in transgenic Brassica napus seeds. Planta 199:515-519

Taji T, Seki M, Satou M, Sakurai T, Kobayashi M, Ishiyama K, Narusaka Y, Narusaka M, Zhu JK, Shinozaki K (2004) Comparative genomics in salt tolerance between Arabidopsis and Arabidopsisrelated halophyte salt cress using Arabidopsis microarray. Plant Physiol 135:1697-1709

Vasekina AV, Yershov PV, Reshetova OS, Tikhonova TV, Lunin VG, Trofimova MS, Babakov AV (2005) Vacuolar $\mathrm{Na}^{+} / \mathrm{H}^{+}$antiporter from barley: Identification and response to salt stress. Biochem Mosc 70:100-107

Wong CE, Li Y, Labbe A, Guevara D, Nuin P, Whitty B, Diaz C, Golding GB, Gray GR, Weretilnyk EA, Griffith M, Moffatt BA (2006) Transcriptional profiling implicates novel interactions between abiotic stress and hormonal responses in Thellungiella, a close relative of Arabidopsis. Plant Physiol 140:1437-1450

Wu CA, Yang GD, Meng QW, Zheng CC (2004) The cotton GhNHX1 gene encoding a novel putative tonoplast $\mathrm{Na}^{+} / \mathrm{H}^{+}$antiporter plays an important role in salt stress. Plant Cell Physiol 45:600-607

Xia T, Apse MP, Aharon GS, Blumwald E (2002) Identification and characterization of a NaCl-inducible vacuolar $\mathrm{Na}^{+} / \mathrm{H}^{+}$antiporter in Beta vulgaris. Physiol Plant 116:206-212

Yamaguchi T, Blumwald E (2005) Developing salt-tolerant crop plants: challenges and opportunities. Trends Plant Sci 10:615620

Yamaguchi T, Fukada-Tanaka S, Inagaki Y, Saito N, YonekuraSakakibara K, Tanaka Y, Kusumi T, Iida S (2001) Genes encoding the vacuolar $\mathrm{Na}^{+} / \mathrm{H}^{+}$exchanger and flower coloration. Plant Cell Physiol 42:451-461

Yamaguchi-Shinozaki K, Shinozaki K (2005) Organization of cis-acting regulatory elements in osmotic- and cold-stress-responsive promoters. Trends Plant Sci 10:88-94

Yamaguchi-Shinozaki K, Shinozaki K (2006) Transcriptional regulatory networks in cellular responses and tolerance to dehydration and cold stresses. Annu Rev Plant Biol 57:781-803

Yang QC, Wu MS, Wang PQ, Kang JM, Zhou XL (2005) Cloning and expression analysis of a vacuolar $\mathrm{Na}^{+} / \mathrm{H}^{+}$antiporter gene from Alfalfa. DNA Seq 16:352-357

Yanhui C, Xiaoyuan Y, Kun H, Meihua L, Jigang L, Zhaofeng G, Zhiqiang L, Yunfei Z, Xiaoxiao W, Xiaoming Q, Yunping S, Li Z, Xiaohui D, Jingchu L, Xing-Wang D, Zhangliang C, Hongya G, Li-Jia Q (2006) The MYB transcription factor superfamily of Arabidopsis: expression analysis and phylogenetic comparison with the rice MYB family. Plant Mol Biol 60:107-124

Yokoi S, Quintero FJ, Cubero B, Ruiz MT, Bressan RA, Hasegawa PM, Pardo JM (2002) Differential expression and function of Arabidopsis thaliana $N H X \mathrm{Na}^{+} / \mathrm{H}^{+}$antiporters in the salt stress response. Plant J 30:529-539

Zhang GH, Su Q, An LJ, Wu S (2008) Characterization and expression of a vacuolar $\mathrm{Na}^{+} / \mathrm{H}^{+}$antiporter gene from the monocot halophyte Aeluropus littoralis. Plant Physiol Biochem 46:117-126

Zhu JK (2001) Plant salt tolerance. Trends Plant Sci 6:66-71

Zhu JK (2002) Salt and drought stress signal transduction in plants. Annu Rev Plant Biol 53:247-273

Zhu JK (2003) Regulation of ion homeostasis under salt stress. Curr Opin Plant Biol 6:441-445 